\title{
El rol docente del tutor y supervisor de prácticas en Trabajo Social: construcción de la reflexividad y el compromiso durante la formación a través de la supervisión
}

\section{Carmina Puig-Cruells}

Licenciada en Trabajo Social y Psicología. Doctora en Antropología

Universidad Rovira i Virgili. Tarragona, España

https://orcid.org/0000-0003-1160-9252 • carmina.puig@urv.cat

\section{Resumen}

Este texto profundiza en temas fundamentales para la formación de los trabajadores sociales y tiene como referencia el artículo "El rol docente del tutor de prácticas y el acompañamiento al estudiante" escrito en 2004. El artículo original lo motivó la necesidad de poner en claro los roles docentes que se ejercen en las prácticas por parte del tutor y del supervisor.

La utilización del artículo referido en el desarrollo de asignaturas de prácticas y supervisión, así como su aportación para entender la complejidad de las relaciones que se establecen durante las prácticas, lo hacen, aun hoy, un texto muy consultado y citado.

Sin embargo, el inevitable y enriquecedor avance del pensamiento, sumado a las modificaciones del contexto social, han ido generando en los últimos años la necesidad de su revisión y ampliación, e incluso su reformulación.

El texto que ahora se presenta contiene elementos conceptuales originales del artículo del 2004, adaptados a la actualidad, al mismo tiempo que se presentan nuevas propuestas de profundización sobre la formación práctica. Se desarrolla y amplía la conceptualización y metodología de la supervisión en el marco de las universidades como un espacio de reflexión y revisión de las prácticas en clave de desarrollo técnico y disciplinar. Al mismo tiempo, se profundiza en los diversos roles y tareas docentes del tutor y del supervisor en el acompañamiento del estudiante. Por todo ello se encontrarán algunos párrafos que necesitan ser entendidos en la época en que fueron escritos, aunque sobre ellos, se avanza con nuevas aportaciones.

Palabras clave: Supervisión; Tutor; Prácticas; Trabajo social.

Recibido: 24/06/2019 | Aprobado: 19/10/2019

(i) (2) Esta obra está bajo una Licencia Creative Commons Atribución-NoComercial-CompartirIgual 4.0 Internacional.

¿Cómo citar este artículo? / How to quote this article?

Puig-Cruells, C. (2020). El rol docente del tutor y supervisor de prácticas en Trabajo Social: construcción de la reflexividad y el compromiso durante la formación a través de la supervisión. Prospectiva. Revista de Trabajo Social e intervención social, (29), 57-72. doi: 10.25100/prts.v0i29.8084. 


\title{
The Teaching Role of the Tutor and Training Supervisor in Social Work: Building Introspection and Commitment During Training via Supervision
}

\begin{abstract}
This article delves deep into the fundamental themes of training for social workers and has as its basis the article "The Teaching Role of the Training Supervisor and Accompaniment of the Student" written in 2004. The original article was written driven by the necessity to clearly define the teaching roles of tutors and supervisors in internship practices.

Its reiterated use in pedagogy, as well as its contributions to understanding the complexity of relationships which are formed during internships, make it, even today, a text which is frequently consulted and cited.

Nevertheless, the inevitable and enriching advance in thought, together with the evolution of social contexts, demand its revision, expansion and even, reformulation.

The text that is currently presented contains some of the original concepts which have been up-dated for relevance today, and at the same time, presents new proposals for a deeper study into practical training. It expands and widens the conceptualization and methodology of supervision within the framework of universities as an area for reflection and review of the practices in terms of both technical and curricular development. The article also explores the various roles in greater depth as well as the teaching tasks of both the tutor and supervisor as they accompany the student.

Although the article makes new contributions to the field, a number of paragraphs will need to be understood with reference to the past, the era in which they were written.

Keywords: Supervision; Tutor; Practical internship; Social work.

Sumario: 1. Introducción, 2. Las prácticas en las enseñanzas de Trabajo Social, 3. La estructura de una relación de aprendizaje durante las prácticas, 4. Objetivos de las prácticas y la relación entre el tutor, el supervisor docente y el estudiante, 5. Los roles del tutor, el supervisor docente y el estudiante, en el marco de una relación integradora en las prácticas, 6. La supervisión durante las prácticas de campo, 7. Expectativas y temores de los estudiantes durante las prácticas y la supervisión, 8. Estrategias de acompañamiento al estudiante desde la supervisión, 9. Conclusiones, 10. Referencias bibliográficas.
\end{abstract}

\footnotetext{
Prospectiva. Revista de Trabajo Social e intervención social • No. $29 \bullet$ ene.-jun. 2020 • pp. 57-72 e-ISSN: 2389-993X • Doi: 10.25100/prts.v0i29.8084

Universidad del Valle - Cali, Colombia
} 
"Nos encontramos ante un saber que nace de la experiencia cuando las ideas y las estrategias que lo estructuran constituyen la forma emergente de una práctica de reflexión en torno a lo vivido. Lo vivido es el acontecer de las cosas que cada cual vive, la experiencia se encuentra allí donde lo vivido va acompañado de pensamiento.

El saber que procede de la experiencia es, por lo tanto, el que se mantiene en una relación pensante con el acontecer de las cosas, el de quien no acepta un estar en el mundo según los criterios de significación dados, sino que va en busca de su propia medida" Eccelli et al., 2002, p. 153.

\section{Introducción}

Las profesiones y organizaciones donde operan los trabajadores sociales tienen unas condiciones sociales y psicológicas, además de responsabilidades y exigencias, sometidas a una mayor complejidad para la intervención social.

Estos fenómenos de complejidad han sido estudiados desde el análisis psicológico o desde la sociología y todas coinciden, como mínimo, en tres factores que inciden en esta cuestión. El primero es la importancia cada vez más creciente de que todos los servicios dirigidos a las personas contribuyen al desarrollo del bienestar psicosocial individual y colectivo. Un segundo factor es la relevancia del factor humano de los profesionales en aquellas tareas y funciones en que es imprescindible el establecimiento de una relación o vínculo. El tercer factor determinante es que la dotación de tecnologías y medios asistenciales no puede sustituir la persona del profesional a la hora de desarrollar la labor de mejorar la calidad de vida y combatir el malestar de las personas.

La tarea profesional que desarrollarán los estudiantes se asienta en la relación que se establece con las personas y apunta mucho más allá de la aplicación de prestaciones o servicios. Los profesionales tratan de ayudar a las personas a afrontar las dificultades en que se encuentran para, junto con ellas, construir o reconstruir su historia. Esta posición de los futuros profesionales genera una mayor complejidad en la formación que se ofrece desde las universidades.

Las profesiones sociales han hecho un gran esfuerzo para conseguir que sus disciplinas se inserten en el campo de las ciencias sociales. Desde diferentes discursos profesionales y académicos ha sido incesante el interés en la capacitación y la formación permanente de los profesionales. Sin embargo, este interés se ha visto a menudo insuficientemente desarrollado en acciones dirigidas a aumentar los análisis sobre la práctica entre las cuales se encuentra la supervisión, que ha tenido un crecimiento muy dispar respecto de otras formaciones.

Una situación similar ha acontecido en la formación práctica de los estudiantes de Trabajo Social. Si bien en los estudios de Trabajo Social se dan un conjunto de actividades prácticas en las que se ejercen funciones propias de los trabajadores sociales en el marco de las instituciones, se debe profundizar más en la supervisión de las prácticas, que no debe ser substituida, tan solo, por seminarios de seguimiento y orientación de las prácticas.

Prospectiva. Revista de Trabajo Social e intervención social • No. $29 \bullet$ ene.-jun. $2020 \bullet$ pp. 57-72 e-ISSN: 2389-993X • Doi: 10.25100/prts.v0i29.8084

Universidad del Valle - Cali, Colombia 
Entre los profesionales, y también entre los estudiantes de Trabajo Social, existe el supuesto de que desarrollar una práctica reflexiva es imprescindible. Se considera que una práctica reflexiva no es una técnica específica, ni un elemento autónomo del oficio, y que no se puede separar de la totalidad de la práctica profesional. Pero la realidad es que a menudo, durante los estudios de grado y posteriormente durante la intervención, hay poco tiempo para pararse a pensar, reflexionar y se especula principalmente con el objetivo de guiar el paso siguiente de la intervención. Estas pequeñas decisiones en el marco de la rutina de las prácticas son tan solo pre-reflexiones que pueden determinar las acciones a realizar, a veces sin suficiente deliberación ni rigor.

El desafío actual se constituye, pues, en facilitar entornos de aprendizaje que aporten herramientas para continuar reflexionando sobre la práctica y aprendiendo de ella de forma permanente, especialmente durante su vida laboral. Como señala Godoy, implica "ubicar al sistema estudiante/docente como una díada comprometida con el aprendizaje, convirtiéndose ambos en agentes activos del proceso formativo y sobre todo del desarrollo de competencias que aseguren que estarán inmersos en espacios de aprendizajes permanentes a lo largo de la vida profesional" (Godoy, 2012, p. 82).

Otra de las características asociada a la formación es el descentramiento que "significa que el saber se sale de los libros y de la escuela" (Barbero, 2003, p. 19). Es decir, la necesidad que tendrán los estudiantes cuando se incorporen al mundo laboral, no tan solo de continuar aprendiendo, sino de continuar reflexionando sobre su práctica porque los saberes se destemporalizan y requieren poder ser revisados, volver a ver, mantenerse atento a las manifestaciones de las personas porque el conocimiento se encuentra en distintos lugares y en distintos tiempos. De aquí la importancia de poner en valor aquellos saberes socialmente útiles, saberes lógicos, simbólicos, históricos y también estéticos.

Este articulo pone en valor el rol docente del tutor de prácticas y el supervisor docente, así como la metodología de la supervisión en el marco de las universidades como un espacio de reflexión y revisión de las prácticas de campo con la finalidad de que la supervisión iniciada durante la formación continúe y se expanda hacia el ejercicio profesional. Además, a esta propuesta se han incorporado las aportaciones de las ciencias psicológicas, sociológicas, la hermenéutica, las teorías de la acción comunicativa, las teorías de las organizaciones y el conjunto de las materias impartidas en la formación académica, así como, los saberes socialmente útiles. Esto ha permitido la integración de un gran número de conceptos y perspectivas disciplinarias.

El artículo se contextualiza en el Trabajo Social, aunque todo lo que se plantea puede ser trasladado a otras disciplinas, y de manera particular a aquellas que se ocupan del trabajo con las personas y a menudo en organizaciones inmersas en la complejidad, en las que conviven procesos de mucha incertidumbre y la emergencia de nuevos fenómenos, donde se genera la necesidad de plantear nuevas perspectivas sobre la acción profesional.

Prospectiva. Revista de Trabajo Social e intervención social • No. 29 • ene.-jun. 2020 • pp. 57-72 e-ISSN: 2389-993X • Doi: 10.25100/prts.v0i29.8084

Universidad del Valle - Cali, Colombia 


\section{Las prácticas en las enseñanzas de Trabajo Social}

Las prácticas en los estudios de Trabajo Social son aquel conjunto de actividades y funciones propias de los trabajadores sociales, que se realizan tanto en los marcos de las instituciones colaboradoras como aquellas que se realizan en las universidades, como son la supervisión y la orientación.

Cuatro ejes serán fundamentales en la experiencia del estudiante durante sus prácticas: la incorporación y posterior desarrollo de actividades en el centro de prácticas, la relación de aprendizaje que se establezca con los tutores, el modelo de programación y evaluación integrador de toda la experiencia, y la supervisión de las prácticas que se realizan en el marco universitario.

La calidad de las prácticas depende en buena medida del tipo de actuaciones y apoyos que se presten a los estudiantes, aunque también depende del nivel de autoconocimiento del estudiante. Si bien es cierto que las prácticas no deben aleccionar al estudiante, conviene que no sean, según Zabalza (2004), "las prácticas desasistidas, des-acompañadas, apenas si poseen virtualidades formativas. Una de las exigencias básicas de un buen practicum es contar con momentos de reposo reflexivo sobre la experiencia que se está viviendo" (p. 9).

\section{La estructura de una relación de aprendizaje durante las prácticas}

La relación de aprendizaje la constituyen tres pilares fundamentales: tiempo, objetivos y relación. La alta dotación de tiempo de prácticas y de espacio de supervisión durante los estudios de Trabajo Social hacen que la relación entre tutor de prácticas, supervisor docente y estudiante se vuelva muy significativa.

Los objetivos de las prácticas consisten esencialmente en iniciar al estudiante en la actividad profesional y crear las condiciones que permitan desarrollar una primera experiencia de profesionalización. Para poder abordarse, deben estructurarse como mínimo en tres áreas: el conocimiento respecto del campo específico de prácticas, el contexto institucional y del servicio, y la tarea que realiza el profesional, los recursos materiales y la documentación.

Un área no menos importante es la del aprendizaje de las actitudes y valores, de la autonomía y de la mejora personal.

Las habilidades y procedimientos también forman parte de los aprendizajes respecto de las relaciones, como la comunicación y las habilidades de escucha de negociación, entre otras. También lo son las técnicas de observación, de entrevista, de manejo de reuniones, de gestión y de documentación, así como las técnicas organizativas, de rendimiento y de cumplimiento. 
Puig-Cruells

\section{Objetivos de las prácticas y la relación entre el tutor, el supervisor docente y el estudiante}

Se considera tutores a los trabajadores sociales que acogen al estudiante en sus lugares de trabajo, sin embargo, los supervisores docentes son profesores adscritos a la Universidad.

La relación entre ambos, incluido el/ la estudiante ha de ser pactada y ha de darse partiendo de aquellos objetivos de conocimiento, actitudes, valores, habilidades y procedimientos que el estudiante debe alcanzar.

Es conveniente que estos objetivos se puedan establecer firmando un acuerdocontrato de prácticas entre el estudiante, el tutor y el supervisor docente. Acuerdo de prácticas donde se establecen cada uno de los objetivos y actividades a realizar, así como los compromisos que asume cada uno de los miembros implicados y respetando los propios límites que existen en cualquier organización. También deben establecerse los compromisos de confidencialidad y acuerdos de supervisión que garanticen un marco de trabajo para todas las partes implicadas.

La negociación de los objetivos de las prácticas debe respetar los objetivos que tienen su origen en las expectativas sobre las prácticas de los tres participantes, así como los recursos disponibles a nivel institucional y/o personales que permitan satisfacer una o más necesidades.

Será el conjunto de esta confrontación entre aspiraciones y recursos lo que deberá señalar cuáles serán los objetivos del plan de prácticas. Solo será posible satisfacer algunas de las aspiraciones. Algunas deberán ser pospuestas y otras renunciadas. Las prioridades de los objetivos a alcanzar están condicionadas por la motivación de quienes deciden, por los valores internalizados y las posibilidades establecidas.

\section{Los roles del tutor, el supervisor docente y el estudiante, en el marco de una relación integradora en las prácticas}

Nos proponemos explorar sobre los roles de los diversos agentes participantes en las prácticas, su relación y los procesos presentes durante su ejercicio docente en las prácticas. Para ello nos hemos de detener en el análisis de los diferentes marcos de relación que se dan en el espacio de las prácticas y la supervisión docente.

Los profesionales tutores tienen un rol docente y juegan un papel fundamental en la formación del estudiante porque ellos son la primera imagen del mundo profesional que recibe el estudiante. Es a través del contacto con el tutor, la presentación del servicio, su manera de trabajar, las prioridades establecidas y su actitud personal, que el estudiante conocerá un estilo y un modelo profesional. Por todos estos motivos se considera importante que los profesionales que ejercen de tutores de prácticas sean conscientes de su rol docente y se interesen en formar a los estudiantes participando de los objetivos pedagógicos del grado.

Prospectiva. Revista de Trabajo Social e intervención social • No. $29 \bullet$ ene.-jun. $2020 \bullet$ pp. 57-72 e-ISSN: 2389-993X・ Doi: 10.25100/prts.v0i29.8084

Universidad del Valle - Cali, Colombia 
El tutor es el profesional que acompaña desde la instrucción y sobre todo desde el modelaje. Los tutores son los referentes educativos más poderosos y eficaces para el estudiante. Su tarea docente es reconocida a través del trabajo pedagógico que realizan con los estudiantes y a través de la atención y del seguimiento de las prácticas. El tutor tiene un rol de orientación in situ, da modelo, y acompaña de forma más próxima la evolución del estudiante. Proporciona soporte y escucha cuando hay deslices del estudiante.

Los tutores realizan un seguimiento del estudiante a través de supervisiones individuales. Este espacio facilita la eficacia de las actividades diarias mediante la organización de la tarea del aprendizaje y el desarrollo práctico de habilidades, el feedback permanente, el apoyo y la contención del estudiante.

La supervisión de los estudiantes por parte del tutor puede darse a dos niveles: el nivel formal, cuando las reuniones entre el tutor y el estudiante están planificadas y con objetivos establecidos, o de tipo informal cuando el tutor orienta y asesora al estudiante sobre las actividades que debe de realizar, sin haber explicitado previamente un encuentro.

La supervisión de tipo individual que realiza el tutor es muy útil al estudiante, ya que le supone recibir un asesoramiento continuado sobre su actuación, le ayuda a ir tomando más responsabilidades en sus prácticas permitiéndole un proceso en el que progresivamente se va haciendo cargo de tareas cada vez más complejas.

El supervisor docente es un profesor adscrito a la Universidad y representa en algunos aspectos un rol más normativo. La supervisión grupal que realiza el supervisor docente revisa las pautas seguidas en las actividades, pide justificaciones sobre lo que se ejercita en las prácticas. Señala y marca, orienta y ofrece asesoramiento sobre aquello considerado conveniente para el desarrollo profesional del alumno. El supervisor docente se aproxima lo máximo posible a lo que sería la supervisión externa en el marco de las instituciones.

El supervisor docente acompaña el proceso de aprendizaje de forma más distante de la acción, pero a su vez posibilita reflexión y volver a repensar y dar significado a la labor y el ejercicio profesional. Su acción pretende analizar las situaciones con rigor, así como relacionar los conocimientos adquiridos y su aplicación en las prácticas. Siguiendo a PuigCruells (2006), se pretende apoyar al estudiante especialmente en el análisis de la realidad compleja donde interviene y donde las relaciones interpersonales son una herramienta fundamental. Permite al estudiante situar su bagaje de conocimientos, técnicas y experiencia de prácticas. Es el lugar idóneo para entender, aprender y organizar la pluralidad de elementos que el estudiante ha aprendido en el aula y en la práctica.

La experiencia práctica siempre genera en el estudiante aspectos personales, respuestas espontáneas, vivencias, sentimientos, contradicciones, inseguridades que el supervisor docente ha de saber valorar y orientar para lograr iniciar al estudiante en intervenciones e implicaciones cada vez más profesionales, descubriendo a la vez los mecanismos a través de los cuales se genera determinada situación.

Prospectiva. Revista de Trabajo Social e intervención social • No. $29 \bullet$ ene.-jun. $2020 \bullet$ pp. 57-72 e-ISSN: 2389-993X・ Doi: 10.25100/prts.v0i29.8084

Universidad del Valle - Cali, Colombia 
La supervisión en grupos de iguales apoya al estudiante en su proceso de prácticas, mediante el análisis y la reflexión de la tarea realizada. Schön (2010) concibe la reflexión a la que nos referimos como una forma de conocimiento, como un análisis y propuesta global que orienta la acción. Así mismo, dicho conocimiento teórico pasa a ser considerado un instrumento de los procesos de reflexión integrándose de forma significativa e imbricándose en los esquemas de pensamiento más genéricos que son activados en la práctica.

También ofrece la formación directa para afrontar las tareas para las cuales el estudiante no se encuentra aún preparado, haciendo referencia a los conocimientos teóricos ya adquiridos. Este marco también posibilita la contención y el soporte emocional delante de las dificultades cotidianas, así como la evaluación del proceso y del rendimiento del estudiante.

Durante el ejercicio de la supervisión, tanto los tutores como los supervisores docentes, tienen que poder mantener un conjunto de características que, siguiendo a Morero y Solano (1996), caracterizan una buena práctica supervisora. Para esta labor tan relevante, los tutores de prácticas y los supervisores docentes deben estar debidamente formados en materia de supervisión y en diversos ámbitos de la intervención social. Ello requiere recibir por parte de las universidades los apoyos y los soportes didácticos necesarios con la finalidad de mantener una buena calidad de atención y enseñanza a los estudiantes. El objetivo es poder desarrollar una práctica basada en la equidad, en la confianza, en el buen dominio de sí mismo, con capacidad abierta a la comprensión de los estudiantes supervisados y con una manifestación clara de sus expectativas para hacerles saber qué se espera de ellos, reconociendo sus méritos y estimulando la buena producción y satisfacción de la tarea.

La experiencia del estudiante durante sus prácticas se sustenta en lo que refiere y recibe a través de los diferentes agentes intervinientes, y se integra de forma singular en el triángulo relacional constituido por él mismo, el tutor durante las prácticas de campo y el supervisor docente de la universidad. El estudiante tiene un rol de aprendizaje, de dejarse conducir, de cumplir con el encargo, aunque tomando iniciativas, de respeto a la autoridad, pero queriendo volar solo.

Cabe decir que ambas actividades de supervisión que se realizan durante el grado, la del tutor y la supervisión del docente, son complementarias, aunque la propia naturaleza de esta relación está revestida de complejidad porque el supervisor docente y el tutor no pertenecen a la misma institución. Sin embargo, tienen un único estudiante al que deben acompañar, contribuir a su desarrollo y establecer buenos vínculos que serán determinantes para el proceso de aprendizaje.

De la forma como los tres participantes puedan establecer objetivos comunes y vínculos para poder establecer marcos de colaboración, dependerá en gran parte el proceso de aprendizaje del estudiante y la satisfacción de ambos formadores. 


\section{La supervisión durante las prácticas de campo}

El espacio de supervisión durante la formación práctica de los estudiantes es un tema apenas investigado. Por este motivo, es nuestro propósito contribuir al conocimiento y al debate sobre los elementos que están presentes en la supervisión de los estudiantes durante las prácticas profesionales, conscientes de que se trata de un tema complejo, puesto que existen muchos posicionamientos y prácticas que persiguen objetivos similares, aunque se ejercen de manera muy distinta. Es en este sentido que nos proponemos sistematizar un conjunto de conceptos y herramientas prácticas que faciliten la innovación en el ámbito de la supervisión de las prácticas de campo.

La experiencia metodológica de nuestra propuesta de supervisión no se limita solo a la organización de espacios académicos de seguimiento de las prácticas cuyo objetivo es poner en común la experiencia realizada. Se propone un acompañamiento desde la supervisión que permita, por una parte, ampliar la gama de informaciones, conocimientos y, por otra, mantener una actividad mental. Como señalan Lanza y Graell (2014), sería un proceso reflexivo que mantenga una dirección deliberada de la acción, una interpretación, un poder dar sentido, empatizar y comprender los estados emocionales o inferir estados mentales que subyacen en las personas que atienden.

Trabajar desde este posicionamiento significa para los estudiantes ser más conscientes de las propias limitaciones y utilizar su propia experiencia, su mentalización, como forma de llegar a comprender los procesos de complejidad variable que se dan en los procesos de atención a las personas, incorporando su experiencia cultural y formativa, y pasando de una apropiación individual a un compartir colectivo en el espacio de supervisión.

Las orientaciones pedagógicas de la supervisión tienen como objetivo revisar y reflexionar sobre el quehacer de las prácticas profesionalizadoras del estudiante y sobre los sentimientos que acompañan la actividad, así como contrastar su experiencia con los marcos teóricos y conceptuales. Consiste, pues, en un espacio de formación y renovación del ejercicio de la profesión para la que se están formando, en el cual cabe hacerse preguntas, dudar. Es un espacio caracterizado por la reflexión sistemática sobre las acciones profesionales que se realizan en el período de prácticas.

La inserción de los estudiantes en un espacio de supervisión desde lo académico implica en cierta medida aceptar el desafío de intentar resolver favorablemente la tensión existente entre las múltiples lógicas, los encargos de la institución donde se realizan las prácticas, las demandas de la sociedad, así como planear y revisar críticamente los enfoques y metodologías pertinentes para abordar los problemas sociales en sus manifestaciones tanto individuales o de grupo, como organizativas.

La supervisión, como nos indican Berasaluze-Correo y Ariño-Altuna (2014), "es el método pedagógico privilegiado para asegurar un adecuado aprendizaje de las prácticas que fomenta la autorreflexión, es decir, posibilita repensar lo vivido, evitando caminos trillados, lugares comunes o estereotipados" (p. 105).

Prospectiva. Revista de Trabajo Social e intervención social • No. $29 \bullet$ ene.-jun. 2020 • pp. 57-72 e-ISSN: 2389-993X・ Doi: 10.25100/prts.v0i29.8084

Universidad del Valle - Cali, Colombia 
Desde esta propuesta, la supervisión académica es entendida como una herramienta pedagógica que se construye en una zona de interfaz entre teoría, método y práctica para la enseñanza-aprendizaje del rol del trabajador social. Tal y como señalan Castellanos, Meschini, y Rampoldi (2015), los estudiantes, cuando supervisan sus intervenciones en lo social, pueden volver a repensar la práctica, así como los marcos ideológico, político, teórico, metodológico y jurídico, donde se inscriben las mismas, alejándose entonces de posiciones estigmatizadoras y moralizantes acerca de lo social.

El espacio de la supervisión se dirigiría, pues, a ser un proceso mediante el cual un estudiante que realiza una práctica recibe la orientación y guía del supervisor docente, de forma que pueda aprovechar lo mejor posible sus conocimientos y habilidades y perfeccionar sus aptitudes con objeto de realizar sus tareas de la forma más eficiente posible. Este acompañamiento permite suministrar un modelo a los estudiantes supervisados y ayuda a poder establecer una relación de congruencia entre las políticas institucionales y los objetivos de las prácticas.

Tres son las características de una buena práctica supervisora según Morera y Solano (1996): la consideración, la facilitación y la participación. La consideración incluye tratar a los estudiantes supervisados como personas con alto potencial, creer en el valor intrínseco de los alumnos y así posibilitar la aprobación necesaria para iniciar el acompañamiento, mostrar interés en los supervisados como personas, manteniendo una comunicación clara con ellos, tratar a los estudiantes con equidad, reconocer sus esfuerzos y estimularlos con anticipación.

La facilitación son aquellas enseñanzas orientadas hacia el trabajo. Se coordinan y dirigen los esfuerzos para alcanzar los objetivos del trabajo. Por lo tanto, es importante brindar recursos técnicos, dando asesoría y apoyo, supervisando muy de cerca, o bien, concentrarse en brindar instrucciones detalladas, accesibles a preguntas y cubriendo necesidades específicas.

La participación se enmarca en el proceso en el que dos o más personas ejercen influencia mutuamente para tomar decisiones que tendrán un impacto sobre ellas mismas en el futuro.

Según Puig-Cruells (2015), la supervisión en el ámbito académico es pues un espacio de trabajo en el cual se aprecia y respeta la progresiva capacitación profesional de los estudiantes, donde se propone un aprendizaje a partir de la propia experiencia, contrastada con los otros miembros del grupo y del supervisor docente, pretende analizar las situaciones con rigor, así como relacionar los conocimientos adquiridos y su aplicación en las prácticas, procura apoyar al estudiante especialmente en el análisis de la realidad compleja donde interviene y donde las relaciones interpersonales son una herramienta fundamental, permite al estudiante situar su bagaje de conocimientos, técnicas y experiencia de las prácticas. Es, pues, el lugar idóneo para entender, aprender y organizar la pluralidad de elementos que el estudiante ha aprendido en el aula y en la práctica.

\footnotetext{
Prospectiva. Revista de Trabajo Social e intervención social • No. $29 \bullet$ ene.-jun. $2020 \bullet$ pp. 57-72 e-ISSN: 2389-993X • Doi: 10.25100/prts.v0i29.8084

Universidad del Valle - Cali, Colombia
} 
Ahora bien, la experiencia práctica siempre genera en el estudiante, aspectos personales, respuestas espontáneas, vivencias, sentimientos, contradicciones, inseguridades que el supervisor ha de saber valorar y orientar para lograr iniciar al estudiante en intervenciones e implicaciones cada vez más profesionales.

La supervisión en grupos de iguales es un gran apoyo para el estudiante en su proceso de prácticas. Es mediante el análisis y la reflexión de la tarea realizada que se posibilita la formación directa del estudiante que le permita afrontar tareas para las cuales aún no se encuentra preparado, trabajando los conocimientos teóricos que dispone y requiriendo la necesaria contención y soporte emocional delante de las dificultades cotidianas. También nos señalan De Vicente y Matulic (2011) que la supervisión contribuye a la construcción de la identidad profesional que es, sin duda, una de las finalidades a destacar en la fase de formación inicial.

Para llevar a término la supervisión se ha de disponer de un tiempo y de un encuadre de trabajo en grupos pequeños de estudiantes $\mathrm{y}$, como ya hemos advertido, con un supervisor debidamente formado en materia de supervisión.

\section{Expectativas y temores de los estudiantes durante las prácticas y la supervisión}

Los estudiantes que acuden a prácticas traen sus expectativas. Éstas pueden ser muy altas o casi inexistentes. La naturaleza de estas depende del juicio que hace el estudiante de su situación. Las expectativas normalmente están teñidas de sentimientos idealizados del tipo: "las prácticas no requerirán esfuerzo", "en el tiempo de prácticas es donde se aprende de verdad", "tan solo es una asignatura", "aquí aprenderé y saldré siendo un profesional", etc. Cuanto más razonables son las expectativas, más posibilidades existen de que los resultados de las prácticas sean más exitosos. La psicoanalista Salzberger-Wittenberg, en su obra La relación asistencial, nos facilitan elementos para una mejor comprensión de los elementos que operan, en cuanto a expectativas y sentimientos del estudiante, en relación con el tutor o supervisor docente de prácticas.

El estudiante, antes de establecer una relación con el tutor o supervisor docente, alberga determinadas esperanzas y expectativas ideales. "Cada nueva empresa tiende a despertar nuestras expectativas ideales (...) es como si dijéramos ahora será diferente, esta persona me dará todo lo que alguna vez desee tener" (Salzberger-Wittenberg, 1980. p. 23). Al mismo tiempo, el estudiante también alberga temores acerca de sí mismo o de la ayuda que le posibilitará el tutor.

Las expectativas del estudiante se expresan en la relación que se establece. Existen diferentes mecanismos que pueden ser utilizados por el estudiante para enfrentarse a sus prácticas:

- El estudiante puede pedir que sea el tutor o el supervisor docente quienes le digan lo que debe hacer como si se tratara de indicaciones $u$ órdenes que son tomadas con resignación. Esta actitud puede originarse en la

Prospectiva. Revista de Trabajo Social e intervención social • No. $29 \bullet$ ene.-jun. $2020 \bullet$ pp. 57-72 e-ISSN: 2389-993X • Doi: 10.25100/prts.v0i29.8084

Universidad del Valle - Cali, Colombia 
ingenuidad e inseguridad que sienten algunos estudiantes al empezar sus prácticas. Si la relación o las indicaciones de los supervisores no toman en consideración estas expectativas, puede que la hostilidad y la incomprensión del estudiante emerjan con gran fuerza.

- El estudiante verbaliza de forma masiva e invasiva las situaciones que le preocupan, sus miedos, a veces también con un exceso de atrevimiento, sin procurar entender qué le pasa, trata de vaciar, de ventilar. El miedo al rechazo o al reproche del tutor o supervisor docente le hace sentirse avergonzado y estos operan como "vaciadores" de la angustia del estudiante.

- $\quad$ El estudiante puede buscar a alguien que le ayude a mejorar e iniciarse en el camino profesional. Si lo que busca es apoyo, compartir los problemas que tiene que abordar y que le ayuden a buscar soluciones, la relación con el tutor y el supervisor docente puede convertirse en muy provechosa y contenedora, ya que el estudiante se siente reconocido, soportado en sobrellevar la ansiedad que le produce su situación de aprendizaje.

- La persona del estudiante necesita ser aceptada tal cual es, con todas sus posibilidades y defectos. Necesita comprensión que a menudo se esfuerza por obtener, aunque tema decepcionar al tutor o al supervisor docente. Es necesario que se mantenga la aceptación incondicional que permita al estudiante enfrentarse a las frustraciones, límites y desilusiones inevitables de todo espacio de aprendizaje.

Los temores son igualmente expectativos y operan sobre la relación. El miedo a no estar a la altura de lo que se les pide, a quedarse en blanco, a no ser competentes, a los fracasos consigo mismo o con su entorno, pueden ocultar información importante al tutor o supervisor o bien desplazar la culpa hacia otros elementos.

Por otra parte, hay estudiantes que no tienen los objetivos claros y se muestran poco motivados. Parece que esperan que algo ajeno a ellos, de carácter mágico, suceda y resuelva su situación. Esperan que el apoyo externo (tutor, supervisor) sustituya por entero su propio auto apoyo, e incluso en ocasiones ya no esperan ni siquiera eso, tan solo asisten a las prácticas.

Algunos estudiantes demandan ser dependientes del tutor o del supervisor docente en el sentido que han articulado su aprendizaje en torno a las múltiples dependencias. Se resisten a plantearse un plan de trabajo sobre sus prácticas que contenga aspectos de todas las áreas establecidas. Aceptan esta situación como un fenómeno natural. Sin embargo también hay estudiantes que luchan por su independencia, disponen de más recursos personales y tienen conciencia de los límites del espacio de prácticas.

Aunque hemos descrito diferentes expectativas y temores en el momento en que un estudiante acude a prácticas, no es nuestra intención ser deterministas ya que ninguna de ellas se dará en estado puro. Más bien pretendemos mostrar que estos diferentes estados 
deben ser tomados en consideración e incorporarlos al conjunto de elementos que han de ser analizados para llevar a cabo la acción docente del tutor y del supervisor docente. Mostrar atención a las expectativas, al momento en que se encuentra el estudiante, posibilita ser más vigilante con las propias expectativas que se depositan sobre el estudiante. Se trata, pues, de poder manejar adecuadamente la propia exigencia con la comprensión y poder vincularse adecuadamente, a la vez que respetar las posibilidades de los agentes participantes. El proceso de aprendizaje, guía y orientación, están sujetos a procesos vitales y humanos llenos de contradicciones, límites e incertidumbres.

\section{Estrategias de acompañamiento al estudiante desde la supervisión}

En el acompañamiento al estudiante desde la supervisión se producen dos tipos de estrategias. La primera consiste en el acompañamiento desde el marco académico y orientado por parte de los supervisores docentes. El estudiante necesita una introducción a la actividad de las prácticas para desarrollar una primera experiencia de profesionalización. Esta introducción a la nueva actividad se puede desarrollar antes de la incorporación a prácticas a modo de entrenamiento en el aula a partir de la elaboración, por parte del estudiante, de una breve historia laboral, de conocimientos y habilidades que pueda poner a disposición del tutor en el primer encuentro formal. Este entrenamiento significa tomar conciencia de donde está, de cuál es su experiencia. Otra actividad complementaria puede ser la elaboración de expectativas y ofertas que el estudiante pueda procurar a la institución que lo acoge en prácticas. El objetivo de esta acción sería conseguir que el estudiante valore sus potencialidades y tome conciencia de sus aspiraciones y anhelos.

La segunda estrategia consiste en el acompañamiento desde el marco de las instituciones colaboradoras y orientado por parte de las tutoras. Los tutores deben estar dotados de instrumentos para conocer más al estudiante. Deben aproximarse a conocer lo que sabe y aquello que desconoce para poder ayudarle, qué experiencia posee, o cuál es su ritmo de trabajo y patrón de aprendizaje. Todas ellas son pautas de gran ayuda en la acción de acompañar un proceso de aprendizaje práctico.

Algunos elementos prácticos para dotarse de conocimiento sobre el estudiante consisten en poder identificar al estudiante lo más ampliamente posible en aquellos aspectos de su situación individual, su educación general y su interés por realizar más formaciones además de la universitaria. Su evolución profesional, sus niveles de independencia económica, sus expectativas profesionales, todos ellos son datos que ayudarán al proceso de tutorización. También es indispensable saber cómo aprende el estudiante. Para ello nos basamos en la psicología del aprendizaje:

- $\quad$ Desde la experiencia. Tiene que realizarlo él mismo para poder aprender.

- Desde la teoría. Puede trasladar lo teórico al campo práctico.

- Desde tomar modelo. Necesita ver cómo lo hace el tutor, después puede imitar, por influencia. Debe haber alguien que lo haga antes, él toma modelo y lo reinterpreta.

Prospectiva. Revista de Trabajo Social e intervención social • No. $29 \bullet$ ene.-jun. $2020 \bullet$ pp. 57-72

e-ISSN: 2389-993X・ Doi: 10.25100/prts.v0i29.8084

Universidad del Valle - Cali, Colombia 
- Desde el estudio. Debe reflexionar y contrastar primero sus ideas con aportaciones documentales. De la experiencia de los demás, asume la experiencia de otros profesionales que le es explicada.

- Desde la búsqueda bibliográfica. Busca la experiencia en los relatos que se exponen en los libros. No es determinante que sean de Trabajo Social.

- Desde la reflexión. Necesita hablar de lo que le ocurre y sentir que existen diferentes opiniones para elegir la suya.

A continuación, se exponen algunos apuntes sobre técnicas que permiten orientar a los diferentes actores implicados, estudiante, tutores y supervisores docentes, con el objetivo de permitir al estudiante que disminuya los temores para centrarse en las prácticas. De esta forma, el estudiante puede tomar perspectiva sobre los cambios que deberá realizar, así como los logros que va a conseguir.

La toma de conciencia por parte del estudiante le permitirá entrar en contacto con sus fuerzas y sus debilidades y recibir gratificaciones de alguien que él respeta. Aumentar la conciencia de la continuidad en el aprendizaje y fortalecer la motivación para aprender y trabajar en la integración de lo aprendido. Al mismo tiempo se desarrolla una autoimagen.

Las técnicas que siguen también permitirán a tutores y supervisores docentes conferirse responsabilidad sobre el estudiante, reconociéndoles status y autoridad, y permitirán desarrollar habilidades profesionales y docentes, así como planificarse el trabajo, reflexionar y evaluar objetivos. Algunas de las técnicas que pueden utilizarse son:

- $\quad$ Ofrecer conocimientos, referencias bibliográficas, nombres de personas a las que consultar. El miedo se da por falta de conocimiento.

- Dar lugar a la palabra, a hablar de progresos y retrocesos. Analizar una situación detenidamente: técnicas de análisis, grabación y registro.

- Encontrar un acuerdo entre el estilo básico del estudiante y la actividad o trabajo a desarrollar. Devolver, es decir, confrontar al estudiante con lo que dice y lo que omite.

- Enseñar técnicas concretas de trabajo: documentación, recursos, teléfono... Ofrecer modelo, enseñando como actuar en directo y después cambiar el rol.

- Entrenamientos previos a la acción. Enseñar a buscar y establecer relaciones entre la experiencia propia y la experiencia de los demás. Aprender a valorarse uno mismo: ¿qué hice? ¿cómo lo hice? Enseñar a trabajar en equipo: escuchar, exponer un problema, analizarlo.

- Criticar constructivamente y en positivo. Criticar es valorar, pedir que aporten materiales y nuevas sugerencias. 


\section{Conclusiones}

La asignatura de prácticas del grado de Trabajo Social ha de permitir al estudiante integrar lo vivido durante su etapa de prácticas para así poder construir una profesionalización reflexiva, imprescindible para abordar la complejidad social actual.

Los roles de los tutores de prácticas y los supervisores docentes son imprescindibles para un aprendizaje de calidad en Trabajo Social. Ambos realizan una supervisión de los estudiantes, aunque con metodologías diferentes.

La relación entre el tutor y el supervisor docente está revestida de complejidades porque ambos pertenecen a diferentes instituciones e intereses, aunque deben acompañar a un único estudiante.

El objetivo de la supervisión persigue una profundización que supera el marco de la puesta en común o seguimiento de la experiencia de prácticas. La supervisión busca establecer un proceso reflexivo que mantenga una dirección deliberada de la acción durante las prácticas.

La supervisión permite a los estudiantes tomar conciencia de sus límites y comprender los procesos sociales complejos, incorporando su experiencia cultural y formativa. Esta les dota de diferentes estrategias para revisar su quehacer durante las prácticas y los sentimientos que les acontecen. Los espacios de supervisión en el marco de la formación permiten que se puedan clarificar expectativas y temores de los estudiantes abriendo posibilidades de autoconocimiento.

La supervisión que se da en el marco de las prácticas de campo y la supervisión profesional durante el ejercicio son un continuo. La supervisión durante la formación universitaria debe continuar posteriormente durante el ejercicio profesional.

Las universidades deben de proporcionar el apoyo y el soporte didáctico necesario a los tutores y supervisores docentes de prácticas con la finalidad de mantener una buena calidad de atención y enseñanza a los estudiantes y así poder desarrollar unas prácticas ajustadas a la complejidad actual de la intervención social.

La supervisión en el campo de las prácticas es la antesala de poder ejercer un Trabajo Social reflexivo y crítico. 


\section{Referencias bibliográficas}

Barbero, J. (2003). Saberes hoy: Diseminaciones, competencias y tranversalidades. Revista Iberoamericana de Educación, (32), 17-34. doi: 10.35362/rie320917.

Berasaluze-Correo, A., y Ariño-Altuna, M. (2014). De la supervisión educativa a la profesional. Cuadernos de Trabajo Social, 27(1), 103-113. doi: 10.5209/rev_CUTS.2014.v27.n1.42464.

Castellanos, B., Meschini, P., y Rampoldi, R. (octubre, 2015). La supervisión académica como forma de re-significar el proceso de enseñanza aprendizaje en el nivel superior de educación. En VIII Jornadas nacionales y 1er. Congreso internacional sobre formación del profesorado. Universidad Nacional de Mar de Plata, Argentina.

De Vicente, I., y Matulic, M.V. (2011). La contribución de la supervisión educativa a la construcción de la identidad profesional en trabajo social. Revista de Servicios Sociales y Política Social, (96), 53-66.

Eccelli, A., Faccincani, C., Longobardi, G., Lusiardi, D., Manenti, E., Migliavacca, F. ... Zamboni, C. (2002). El perfume de la maestra. En los laboratorios de la vida cotidiana. España: Icaria.

Godoy, W. (2012). La práctica en los procesos formativos de los y las trabajadores sociales: más allá de la reproducción. Revista Perspectivas, (23), 79-95. doi: 10.29344/07171714.23.464.

Lanza, G., y Graell, A. (2014). Mentalización, apego y regulación emocional. Revista Desnvolupa, (36), 1-16. Recuperado de http://www.desenvolupa.net/Ultimsarticles/Mentalizacion-apego-y-regulacion-emocional.-Angelina-Graell-GustavoLanza-10-2014.

Morera, N., y Solano, A. (1996). Proceso de supervisión y perfil del supervisor de las prácticas académicas del nivel de licenciatura. Recuperado de http://www.ts.ucr.ac.cr/binarios/docente/pd-000059.pdf.

Puig-Cruells, C. (2006). La profesionalización del estudiante y el espacio practico de calidad. Plan de prácticum de intervención e innovación docente: el rol docente del tutor de práctica. El acompañamiento del estudiante. Formación y supervisión. Acciones e Investigaciones Sociales, (1ext.), 311-323. doi: 10.26754/ojs_ais/ais.20061\%20Ext412.

Puig-Cruells, C. (2015). La supervisión en la acción social. Una oportunidad para el bienestar de los profesionales. Tarragona, España: Publicaciones URV. Recuperado de http://llibres.urv.cat/index.php/purv/catalog/download/211/247/4851?inline $=1$.

Salzberger-Wittenberg, I. (1980). La relación asistencial. Aportes del psicoanálisis kleiniano. Buenos Aires. Argentina: Amorrutu editores.

Schön, D. (2010). La formación de profesionales reflexivos. Barcelona, España: Paidós.

Zabalza, M. (2004). Condiciones para el desarrollo del practicum. Profesorado, revista de currículum y formación del profesorado, 8(2), 1-22. 


\section{OTROS ARTÍCULOS DE PROSPECTIVA No. 29 DE 2020}

EDITORIAL

La Reconceptualización: una opción a la encrucijada del Trabajo Social en Colombia Víctor Mario Estrada-Ospina

\section{ARTÍCULOS}

Formación profesional y la práctica supervisada en el Servicio Social brasileño

Reginaldo Ghiraldelli

El rol docente del tutor y supervisor de prácticas de Trabajo Social: construcción de la reflexividad y el compromiso durante la formación a través de la supervisión

Carmina Puig-Cruells

Problematizando las prácticas preprofesionales en Trabajo Social. Desafios y perspectivas

Ruth Noemí Parola

Trabajo Social en contextos de formación política: la práctica transformadora

Angélica López-Yepes

Jackeline Cuenca-Echeverry

Investigación cuantitativa en una práctica universitaria de intervención en comunidad en el norte de México

Adán Cano-Aguilar
Interventores y comunidades vulnerables: una propuesta de formación para universitarios

Dulce Nayeli Cohetero-Hernández

Fidel González-Quiñones

Reagrupación familiar de menores en Aragón. Propuestas de acompañamiento en el proceso migratorio

Chabier Gimeno-Monterde

José David Gutiérrez-Sánchez

Aportaciones de la Educación en Derechos Humanos (EDH) y la Investigación Acción Participativa (IAP) en contextos de trabajo comunitario

Carla Cubillos-Vega

Condiciones para una NeoReconceptualización del Trabajo Social en Chile, Latinoamérica y el Caribe

Luis Vivero-Arriagada

Justicia sin daño: una apuesta por el fortalecimiento del acceso a la justicia Diego Fernando Acosta-Daza

\section{IN MEMORIAM}

Camilo Bautista Ochoa, un santandereano que se enamoró del Pacífico

Jesús Glay Mejía-Naranjo

\section{PROSPECTIVA}

Revista de Trabajo Social e Intervención Social 